\title{
Recognizing Face or Object from a Single Image: Linear vs. Kernel Methods on 2D Patterns
}

\author{
Daoqiang Zhang ${ }^{1,2}$, Songcan Chen ${ }^{1}$, and Zhi-Hua Zhou ${ }^{2}$ \\ ${ }^{1}$ Department of Computer Science and Engineering \\ Nanjing University of Aeronautics and Astronautics, \\ Nanjing 210016, China \\ \{dqzhang, s.chen\} @nuaa. edu.cn \\ ${ }^{2}$ National Laboratory for Novel Software Technology \\ Nanjing University, Nanjing 210093, China \\ zhouzh@nju.edu.cn
}

\begin{abstract}
We consider the problem of recognizing face or object when only single training image per class is available, which is typically encountered in law enforcement, passport or identification card verification, etc. In such cases, many discriminant subspace methods such as Linear Discriminant Analysis (LDA) fail because of the non-existence of intra-class variation. In this paper, we propose a novel framework called 2-Dimensional Kernel PCA (2D-KPCA) for face or object recognition from a single image. In contrast to conventional KPCA, 2D-KPCA is based on 2D image matrices and hence can effectively utilize the intrinsic spatial structure information of the images. On the other hand, in contrast to 2D-PCA, 2D-KPCA is capable of capturing part of the higher-order statistics information. Moreover, this paper reveals that the current 2D-PCA algorithm and its many variants consider only the row information or column information, which has not fully exploited the information contained in the image matrices. So, besides proposing the unilateral 2D-KPCA, this paper also proposes the bilateral 2D-KPCA which could exploit more information concealed in the image matrices Furthermore, some approximation techniques are developed for improving the computational efficiency. Experimental results on the FERET face database and the COIL-20 object database show that: 1) the performance of KPCA is not necessarily better than that of PCA; 2) 2D-KPCA almost always outperforms 2D-PCA significantly; 3) the kernel methods are more appropriate on 2D pattern than on $1 \mathrm{D}$ patterns.
\end{abstract}

\section{Introduction}

Face and object recognition have been an active research area of computer vision and pattern recognition for decades, and many powerful recognition algorithms have been proposed [16]. Among them, subspace methods such as principal component analysis (PCA) [8], linear discriminant analysis (LDA) [11][2] and Bayesian algorithm [4] have been extensively studied and many variants of them have been proposed [9][16]. Recently, the popular 'kernel trick' [7][11] and matrix-based (or more generally, tensor-based) representation of faces or objects without image-to-vector transformation [12][13][15][2] have been introduced into subspace based face recognition, and 
accordingly, the so-called kernel PCA (KPCA) [7], kernel LDA (KLDA) [11], 2DPCA [12] and 2DLDA [2] have been proposed independently.

In some specific scenarios such as law enforcement, passport or identification card verification, etc, there may be only single image per class can be used for training the face recognition system. This brings great trouble to many existing algorithms such as LDA and Bayesian algorithm, which require at least two training samples per class to obtain the so-needed intra-class variation. we only consider PCA and its variant in this paper for face or object recognition from a single image. In [10], a method called $(\mathrm{PC})^{2} \mathrm{~A}$ was proposed as an extension of the standard PCA, which combines the original face image with its first-order projected image and then performs PCA on the enriched version of the image. In [1], the enhanced (PC) ${ }^{2} \mathrm{~A}$ was proposed to use higher order projected images. In [14], the singular value decomposition (SVD) was adopted to generate virtual samples and then perform PCA on the combined images. In [3], a probabilistic approach was described, in which the model parameters were estimated by using a set of images generated around a so-called representative sample image.

As mentioned above, KPCA and 2DPCA are two important variants of PCA based on the kernel trick and matrix-based image representation respectively. The basic idea of kernel trick is to perform the linear analysis by nonlinearly transforming the original input space into a higher or even infinite dimensional feature space and expect that the nonlinear problems in original space can be converted into a linear one in the transformed space. On the other hand, the key idea of 2DPCA is to represent images as matrices without image-to-vector transformation and expect to utilize the underlying spatial structure information for efficient feature extraction and recognition. Although KPCA and 2DPCA have been successfully used for face and object recognition with multiple images per class, their performance evaluation on single image per class remains unknown.

In this paper, a novel framework called 2D Kernel PCA (2D-KPCA) is first proposed, which integrates both advantages of KPCA and 2D-PCA. In contrast to KPCA, 2D-KPCA is based on 2D image matrices and hence can effectively utilize the intrinsic spatial structure information of the images, which is ignored in traditional KPCA after the image-to-vector transformation. On the other hand, in contrast to 2D-PCA, 2D-KPCA is capable of capturing part of the higher-order statistics information, while the linear 2D-PCA can address at most the second order statistics. Moreover, this paper reveals that the current 2D-PCA algorithm and its many variants consider only the row information or column information, which has not fully exploited the information contained in the image matrices. So, besides proposing the unilateral 2DKPCA, this paper also proposes the bilateral 2D-KPCA which could exploit more information concealed in the image matrices Furthermore, some approximation techniques are developed for improving the computational efficiency. Then a comparative study is made on performances of the above four methods on recognizing the face and object from a single image. Experiments are carried out on two well-known databases: the partial FERET face database [6] and the COIL-20 object database [5]. The results show that when recognizing the face and object from only a single image: 1) the performance of KPCA is not necessarily better than that of PCA (in fact if without kernel parameters optimization, KPCA is inferior to PCA in most cases in our experiments); 2) 2D-KPCA nearly always outperforms 2D-PCA significantly; 3) the kernel methods are more appropriate on $2 \mathrm{D}$ pattern than on $1 \mathrm{D}$ patterns. 
The rest of this paper is organized as follows: In Section 2, we present the 2DKPCA framework. Section 3 gives the experimental results on partial FERET face database and COIL-20 object database. Finally, we conclude in Section 4.

\section{Two-Dimensional Kernel PCA}

\subsection{Unilateral 2D-KPCA}

Given $M$ training face or object images, denoted by $m$ by $n$ matrices $A_{k}(k=1,2, \ldots M)$. In traditional KPCA, a kernel-induced mapping function maps the data vector from original input space to a higher or even infinite dimensional feature space. Define the kernel mapping on matrices as

$$
\Phi(A)=\left[\phi\left(A^{1}\right)^{T}, \phi\left(A^{2}\right)^{T}, \ldots, \phi\left(A^{m}\right)^{T}\right]^{T}
$$

where $A^{i}$ is the $i$-th row vector $(1$ by $n$ ) of the matrix $A$ and $\phi$ is conventional kernel mapping on vectors. Let $S_{t}=\sum_{K=1}^{M}\left(\Phi\left(A_{k}\right)-\bar{\Phi}\right)^{T}\left(\Phi\left(A_{k}\right)-\bar{\Phi}\right)$, here $\bar{\Phi}=1 / M \sum_{k=1}^{M} \Phi\left(A_{k}\right)$. Without loss of generality, assume that $\bar{\Phi}=0$, then

$$
S_{t}=\sum_{K=1}^{M} \Phi\left(A_{k}\right)^{T} \Phi\left(A_{k}\right)=\sum_{K=1}^{M} \sum_{i=1}^{m} \phi\left(A_{k}^{i}\right)^{T} \phi\left(A_{k}^{i}\right) \triangleq \Phi^{T} \Phi
$$

here $\Phi=\left[\phi\left(A_{1}^{1}\right)^{T}, \ldots, \phi\left(A_{1}^{m}\right)^{T}, \ldots, \phi\left(A_{M}^{1}\right)^{T}, \ldots, \phi\left(A_{M}^{m}\right)^{T}\right]^{T}$.

In Unilateral 2D-KPCA (denoted as U2D-KPCA), the following criterion is adopted to compute the optimal projective vector $v$

$$
J(v)=\operatorname{trace}\left(v^{T} S_{t} v\right)=v^{T} \Phi^{T} \Phi v
$$

which is equivalent to solve the eigenvalue problem: find $\lambda \geq 0$ and eigenvectors $v \in \operatorname{span}\left\{\phi\left(A_{k}^{i}\right)^{T}, i=1, \ldots, m ; k=1, \ldots, M\right\}$, satisfying $\lambda v=\Phi^{T} \Phi v$.

If we follow the conventional kernel analysis as in KPCA, there exist $m M$ samples to span the kernel feature space $\left\{\phi\left(A_{k}^{i}\right)^{T}, i=1, \ldots, m ; k=1, \ldots, M\right\}$, which will result in heavy computational cost for subsequent optimization procedure. To alleviate the computational cost, in this paper, we use $M$ samples to approximate the kernel feature space: $\tilde{\Phi}=\left[\phi\left(\bar{A}_{1}\right)^{T}, \ldots, \phi\left(\bar{A}_{M}\right)^{T}\right]^{T}$, here $\bar{A}_{k}$ is the mean of the $m$ row vectors of $A_{k}$. So $v=\tilde{\Phi}^{T} q$, and we have the following equivalent problem

$$
\lambda K_{m} q=K^{T} K q
$$

where $K_{m}=\tilde{\Phi} \tilde{\Phi}^{T}$ is the $M$ by $M$ kernel matrix and $K=\Phi \tilde{\Phi}^{T}$ is the $M m$ by $M$ kernel matrix. 
Suppose $R=\left[q_{1}, q_{2}, \ldots, q_{d}\right] \in \Re^{M \times d}$ are the solutions of Eq. (4) corresponding to the largest $d$ eigenvalues, then $v_{i}=\tilde{\Phi}^{T} q_{i}, i=1, \ldots, d$ is the solutions of Eq. (3). For extracting features for a new pattern $A \in \mathfrak{R}^{m \times n}$ with unilateral 2D-KPCA, one simply projects the mapped pattern $\Phi(A)$ onto $v_{1}, \ldots, v_{d}$

$$
Y=\Phi(A)\left[v_{1}, \ldots, v_{d}\right]=\Phi(A) \tilde{\Phi}^{T} R=K_{\text {row }} R
$$

Here $K_{\text {row }}$ is the $m$ by $M$ kernel matrix, and $Y$ is the extracted $m$ by $d$ feature matrix .

The essence of aforementioned U2D-KPCA can be seen as performing conventional KPCA on the rows of the image matrices when each row is treated as an individual element. Similarly, we can construct the alternative U2D-KPCA if treating each column of images as an individual element.

Denote $\Phi(A)=\left[\phi\left(A^{1}\right), \phi\left(A^{2}\right), \ldots, \phi\left(A^{n}\right)\right]$, where $A^{i}$ is the $i$-th column vector $(m$ by 1 ) of the matrix $A$, then

$$
S_{t}=\sum_{K=1}^{M} \Phi\left(A_{k}\right) \Phi\left(A_{k}\right)^{T}=\sum_{K=1}^{M} \sum_{i=1}^{n} \phi\left(A_{k}^{i}\right) \phi\left(A_{k}^{i}\right)^{T} \triangleq \Phi \Phi^{T}
$$

Here $\Phi=\left[\phi\left(A_{1}^{1}\right), \ldots, \phi\left(A_{1}^{n}\right), \ldots, \phi\left(A_{M}^{1}\right), \ldots, \phi\left(A_{M}^{n}\right)\right]$.

The objective function for alternative U2D-KPCA is

$$
J(v)=\operatorname{trace}\left(v^{T} S_{t} v\right)=v^{T} \Phi \Phi^{T} v
$$

Let $\tilde{\Phi}=\left[\phi\left(\bar{A}_{1}\right), \ldots, \phi\left(\bar{A}_{M}\right)\right]$, here $\bar{A}_{k}$ is the mean of the $n$ column vectors of $A_{k}$, so $v=\tilde{\Phi} \alpha$, and we have the following equivalent problem

$$
\lambda K_{m} \alpha=K K^{T} \alpha
$$

where $K_{m}=\tilde{\Phi}^{T} \tilde{\Phi}$ is the $M$ by $M$ kernel matrix and $K=\tilde{\Phi}^{T} \Phi$ is the $M$ by $M n$ kernel matrix.

Suppose $L=\left[\alpha_{1}, \alpha_{2}, \ldots, \alpha_{d}\right] \in \Re^{M \times d}$ are the solutions of Eq. (8) corresponding to the largest $d$ eigenvalues, then $v_{i}=\tilde{\Phi} \alpha_{i}, i=1, \ldots, d$ is the solutions of Eq. (7). For extracting features for a new pattern $A \in \mathfrak{R}^{m \times n}$ with alternative U2D-KPCA, one simply projects the mapped pattern $\Phi(A)$ onto $v_{1}, \ldots, v_{d}$

$$
Z=\left[v_{1}, \ldots, v_{d}\right]^{T} \Phi(A)=L^{T} \tilde{\Phi}^{T} \Phi(A)=L^{T} K_{c o l}
$$

Here $K_{c o l}$ is the $M$ by $n$ kernel matrix, and $Z$ is the extracted $d$ by $n$ feature matrix.

\subsection{Bilateral 2D-KPCA}

As analyzed above, U2D-KPCA and alternative U2D-KPCA are essentially KPCA on rows and columns of images respectively. However, both U2D-KPCA and alternative U2D-KPCA only consider the dependency (correlation) among the row or column 
vectors of the image matrix and neglects the other one. Therefore, some useful information for recognition may be lost in them. Considering this, the bilateral 2D-KPCA is proposed by integrating U2D-KPCA (Eq. (5)) and alternative U2D-KPCA (Eq. (9)) together, which could exploit more information concealed in the image matrices.

After performing U2D-KPCA (Eq. (5)) and alternative U2D-KPCA (Eq. (9)), $m$ by $d$ feature matrix $Y$ and $d$ by $n$ feature matrix $Z$ are obtained for each image. They are combined together for recognition. In this paper, we propose two ways for combining feature matrices $Y$ and $Z$. In the first bilateral 2D-KPCA method (denoted as B2DKPCA-1), $Y$ and $Z$ are firstly transformed into $1 \mathrm{D}$ vectors independently for each images, and then PCA is applied onto these vectors ( $Y \mathrm{~s}$ and $Z s$ ) respectively. Finally, two shorter vectors are further combined into one vector for classification. In the second bilateral 2D-KPCA method (denoted as B2D-KPCA-2), $Y$ and $Z$ are firstly transformed into $1 \mathrm{D}$ vectors and then combined into one $1 \mathrm{D}$ vectors for each images, and then perform PCA on the combined vectors.

It is worthy noting that for comparison, we also implemented the bilateral 2D-PCA algorithms (denoted as B2D-PCA-1 and B2D-PCA-2 respectively) according to a similar procedure as 2D-KPCA. And accordingly, the unilateral 2D-PCA introduced in Section 2 is denoted as U2D-PCA.

\section{Experimental Results}

In this section, a series of experiments are presented to evaluate the performances of the proposed 2D-KPCA including U2D-KPCA, B2D-KPCA-1 and B2D-KPCA-2, compared with existing PCA, KPCA and 2D-PCA methods on single training image per class recognition. These algorithms are tested on two well-known datasets, FERET face database [6] and COIL-20 object database [5]. In our experiments, we adopted the Gaussian kernel function: $k(x, y)=\exp \left(\frac{\|x-y\|^{2}}{2 \sigma^{2}}\right)$ for KPCA and 2D-

KPCA, and kernel width $\sigma$ are chosen as the standard variation of training data. It is worthy noting that for fair comparison, we don't perform any kernel or parameters optimization for both KPCA and 2D-KPCA. And in all the experiments, the nearest neighbor classifier is employed for classification.

\subsection{FERET Face Database}

In this experiment, a partial FERET face database containing 400 gray-level frontal view face images from 200 persons are used, each of which is cropped with the size of $60 \times 60$. There are 71 females and 129 males; each person has two images (fa and fb) with different facial expressions. The fa images are used as gallery for training while the fb images as probes for test.

Figure 1 gives the comparisons of accuracies of linear and kernel methods under different feature dimensions on FERET face database. Here total 4 pairs of methods are compared: (a) PCA vs. KPCA; (b) U2D-PCA vs. U2D-KPCA; (c) B2D-PCA-1 vs. B2D-KPCA-1; (d) B2D-PCA-2 vs. B2D-KPCA-2. It can be seen from Fig. 1 that except KPCA, the other three kernel methods outperform the corresponding linear methods greatly. Table 1 gives comparison of accuracies of linear and kernel methods 
on FERET database, including results of three recent methods for single image face recognition on the same database. And Table1 also shows that except KPCA, the other three kernel methods proposed in this paper outperforms much better than the corresponding linear methods.

Then why the kernel methods perform better on 2D patterns than on $1 \mathrm{D}$ patterns? We guess one reason maybe that the $2 \mathrm{D}$ representations in some sense enlarge the size of samples through treating each rows or columns of images as individual samples, and hence the image covariance matrix in kernel-induce feature space is more accurately evaluated than in $1 \mathrm{D}$ representation where each class has only single sample.
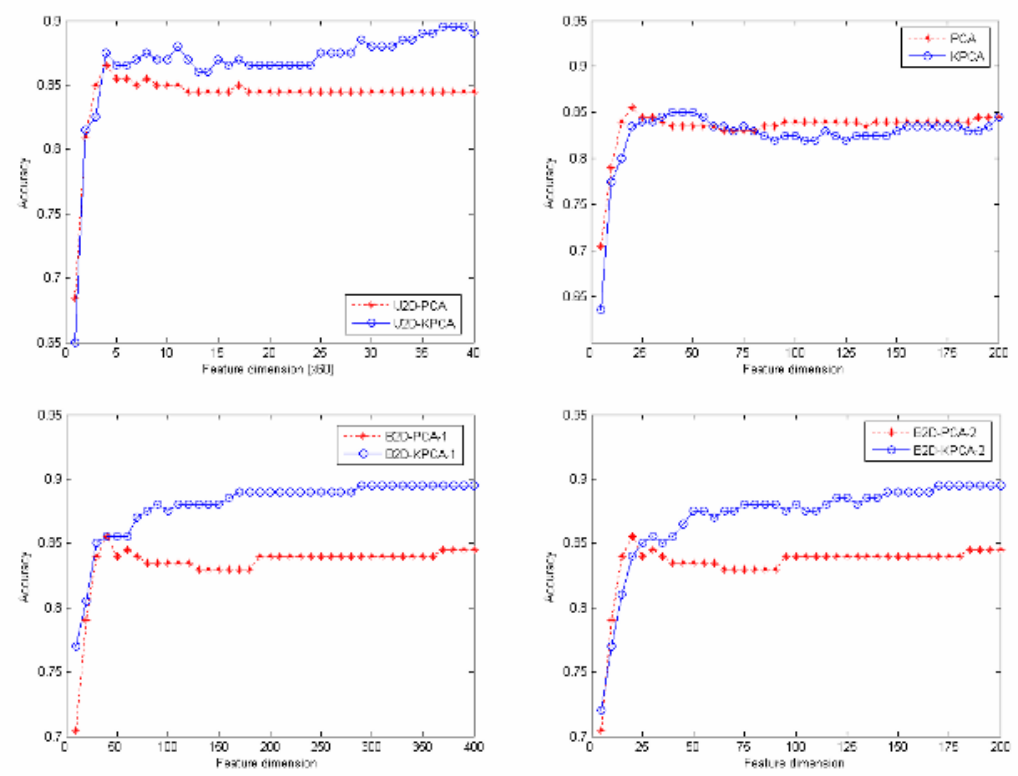

Fig. 1. Comparisons of accuracies of linear and kernel methods under different feature dimensions on FERET face database

\subsection{COIL-20 Object Database}

COIL-20 is a database of gray-scale images of 20 objects. The objects were placed on a motorized turntable against a black background. The turntable was rotated through 360 degrees to vary object pose with respect to a fixed camera. Images of the objects were taken at pose intervals of 5 degrees, which corresponds to 72 images per object. In our experiments, each of the 1440 images were cropped with the size of $64 \times 64$. For each of the 20 objects, we only use the first image per object as the training image, and the rest 71 images for testing.

Figure 2 gives comparisons of accuracies of linear and kernel methods under different feature dimensions on COIL-20 object database. Figure 2 shows that the performance of KPCA is not necessarily better than that of PCA. In fact, KPCA is inferior to PCA in most cases in this experiment. On the other hand, it can be also 
seen from Fig. 2 that the proposed three kernel methods nearly always outperform the corresponding linear methods on this database.

Table 2 gives the detailed comparisons of the relative recognition ability between linear and kernel methods. For each of the 20 objects, the first image is used for training, and the rest 71 images for testing. On each of the 71 images, if the accuracy of the kernel method is higher than that of corresponding linear method, then the count of 'win' plus 1 , and vice versa. Then the counts are averaged on the 20 objects and different dimensions. Table 2 indicates that except KPCA, the performances of the other three kernel methods are better than corresponding linear ones.
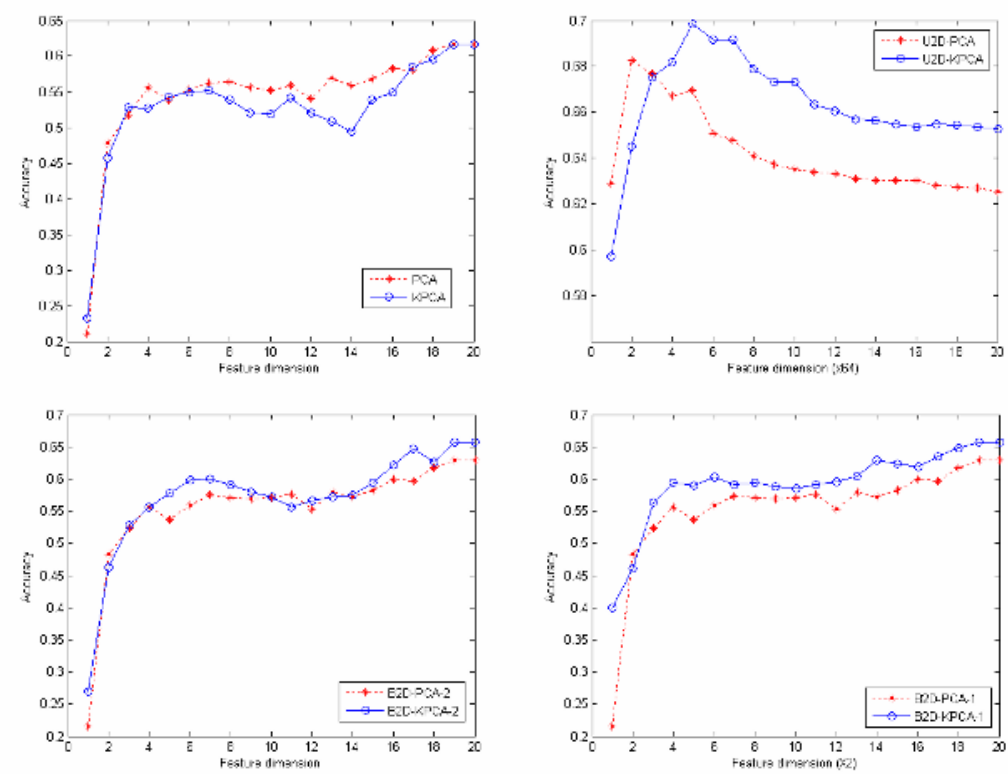

Fig. 2. Comparisons of accuracies of linear and kernel methods under different feature dimensions on COIL-20 object database

Table 1. Comparisons of accuracies of linear and kernel methods on FERET face database

\begin{tabular}{ccc}
\hline & Method & Accuracy (\%) \\
\hline \multirow{6}{*}{ Linear } & PCA [1] & 83.0 \\
& (PC) ${ }^{2}$ A [10] & 83.5 \\
& E(PC) ${ }^{2}$ A [1] & 85.5 \\
& (2D) $)^{2}$ PCA [15] & 85.0 \\
& U2D-PCA & 84.5 \\
& B2D-PCA-1 & 84.5 \\
& B2D-PCA-2 & 84.5 \\
\hline \multirow{5}{*}{ Kernel } & KPCA & 83.5 \\
& U2D-KPCA & 89.0 \\
& B2D-KPCA-1 & 89.5 \\
& B2D-KPCA-2 & 89.5 \\
\hline
\end{tabular}


Table 2. Comparisons of relative recognition ability between linear and kernel methods on COIL-20 object database

\begin{tabular}{cccc}
\hline Match & win & $\begin{array}{l}\text { stand- } \\
\text { off }\end{array}$ & lose \\
\hline $\begin{array}{c}\text { KPCA vs. PCA } \\
\text { U2D-KPCA vs. }\end{array}$ & 11.3 & 32.6 & 27.1 \\
U2D-PCA & 33.5 & 16.6 & 20.9 \\
$\begin{array}{c}\text { B2D-KPCA-1 } \\
\text { vs. B2D-PCA-1 }\end{array}$ & 34.1 & 17.7 & 19.2 \\
$\begin{array}{c}\text { B2D-KPCA-2 } \\
\text { vs. B2D-PCA-2 }\end{array}$ & 28.3 & 19.2 & 23.5 \\
\hline
\end{tabular}

\section{Conclusions}

In this paper, we propose a novel framework called 2D-KPCA (including U2DKPCA, B2D-KPCA-1 and B2D-KPCA-2) for face and object recognition from a single image. Then we make a comparative study on performances of the linear and kernel methods on recognizing the face and object from a single image on two wellknown databases: the partial FERET face database and the COIL-20 object database. The experimental results suggest that, when recognizing the face and object from only a single image: 1) the performance of KPCA is not necessarily better than that of PCA; 2) 2D-KPCA nearly always outperforms 2D-PCA significantly; 3) the kernel methods are more appropriate on 2D pattern than on $1 \mathrm{D}$ patterns.

\section{Acknowledgements}

This work was supported by NSFC (60505004, 60473035), JiangsuSF (BK2004001, BK2005122), the Jiangsu Postdoctoral Research Foundation, and funds from Shanghai Key Laboratory of Intelligent Information Processing. Portions of the research in this paper use the FERET database of facial images collected under the FERET program.

\section{References}

1. Chen, S.C, Zhang, D.Q., Zhou, Z.-H.: Enhanced (PC)2A for face recognition with one training image per person. Pattern Recognition Letters, 25 (2004) 1173-1181

2. Kong, H., Wang, L., Teoh, E.K., Wang, J.G., Venkateswarlu, R.: A framework of 2D Fisher discriminant analysis: applications to face recognition with small number of training samples. In: IEEE Conf. CVPR, 2005

3. Martinez, A.M.: Recognition imprecisely localized, partially occluded, and expression variant faces from a single sample per class. IEEE Trans. on Pattern Analysis and Machine Intelligence, 25(6) (2002) 748-763 
4. Moghaddam, B., Jebara, T., Pentland, A.: Bayesian face recognition. Pattern Recognition 33(11) (2000) 1771-1782

5. Nene, S.A., Nayar, S.K., Murase, H.: Columbia Object Image Library (COIL-20). Technical Report CUCS-005-96, February 1996

6. Phillips, P.J., Wechsler, H., Huang, J., Rauss, P.J.: The FERET database and evaluation procedure for face-recognition algorithms. Image and Vision Computing, 16 (5) (1998) 295-306

7. Scholkopf, B., Smola, A., Muller K.: Nonlinear Component Analysis as a Kernel Eigenvalue Problem. Neural Computation 10(5) (1998) 1299-1319

8. Turk, M., Pentland, A.: Eigenfaces for recognition. J. Cognitive Neuroscience, 3 (1) (1991) 71-86

9. Wang, X., Tang, X.: A unified framework for subspace face recognition. IEEE Trans. on Pattern Analysis and Machine Intelligence, 26(9) (2004) 1222-1228

10. Wu, J., Zhou, Z.-H: Face Recognition with one training image per person. Pattern Recognition Letters 23(14) (2002) 1711-1719

11. Yan, S.C, Xu, D., Zhang, L., Zhang, B.Y., Zhang, H.J.: Coupled kernel-based subspace learning. In: IEEE Conf. CVPR, 2005

12. Yang, J., Zhang, D., Frangi, Yang, J.Y.: Two-dimensional PCA: a new approach to appearance-based face representation and recognition. IEEE Trans. on Pattern Analysis and Machine Intelligence, 26 (1) (2004) 131-137

13. Zhang, D.Q., Chen, S.C., Liu, J.: Representing image matrices: Eigenimages vs. Eigenvectors. In: Proceedings of the 2st International Symposium on Neural Networks (ISNN'05), 659-664, Chongqing, China, 2005

14. Zhang, D.Q., Chen, S.C., Zhou, Z.-H.: A new face recognition method based on SVD perturbation for single example image per person. Applied Mathematics and Computation, 163(2) (2005): 895-907

15. Zhang, D.Q., Zhou, Z.-H.: (2D)2PCA: 2-directional 2-dimensional PCA for efficient face representation and recognition. Neurocomputing, 69(1-3) (2005): 224-231.

16. Zhao, W., Chellappa, R., Rosenfeld, R., Phillips, P.J.: Face recognition: a literature survey. [http://citeseer.n j.nec.com/374297.html], 2000 